\title{
COVID Booster Shot and the Third Dose
}

\section{Hazim Abdul Rahman Alhit*}

Head of Emergency Faculty in Higher Health Institute, General Surgeon Specialist M.D, Al Anbar Directorate of Health, Iraq

Correspondence to: Hazim Abdul Rahman Alhiti, Head of Emergency Faculty in Higher Health Institute, General Surgeon Specialist M.D, Al Anbar Directorate of Health, Iraq.

Received date: December 17, 2021; Accepted date: December 24, 2021; Published date: December 31, 2021

Citation: Alhiti HAR (2021) COVID Booster Shot and the Third Dose. J Med Res Surg 2(6): pp. 1. doi: 10.52916/jmrs214065

Copyright: (C2021 Alhiti HAR. This is an open-access article distributed under the terms of the Creative Commons Attribution License; which permits unrestricted use; distribution and reproduction in any medium; provided the original author and source are credited.

\section{Introduction}

The protection provided by the original shot(s) has begun to decrease over time, then a Coronavirus Disease (COVID) booster shot is given. Moreover, the immunity from the initial dose(s) naturally starts to wane, and you would get a booster. Accordingly, the booster is designed in order to maintain the people's level of immunity for longer [1].

There is evidence that the effect of the vaccine wanes over time, so the officials recommended booster shots for everyone who is eighteen and older [2]. Therefore, the medics instructed the third dose for individuals who are immuno-compromised. The third dose is needed when a person's immune response likely has not responded fully to the vaccine after the first doses. Subsequently, the Food and Drug Administration (FDA) counseled that the EUAs of the Moderna and Pfizer vaccines boost people who are older than eighteen. Moreover, FDA instructed Pfizer third boosters for persons older than sixteen [3].

Accordingly, the reader is eligible for a COVID-19 booster if your age is 18 or older and received the Janssen/Johnson \& Johnson vaccine at least two months ago, 18 or older, and received both required doses of the Moderna vaccine at least six months ago and 16 or older and received both doses of the Pfizer-BioNTech vaccine at least six months ago [1]. Hereafter, the Centers for Disease Control and Prevention (CDC) and the FDA suggested the third dose of a COVID-19 vaccine for patients on cancer treatment, patients after an organ transplant, patients on immune suppressant drugs, patients on stem cell transplant, patients who have primary immunodeficiency, patients have Human Immunodeficiency Virus (HIV) disease, patients on steroids or other medications that may cause severe suppression of the immune system [2].

After any person gets a COVID booster, he might encounter some transient symptoms such as a sore, swollen injected area. Furthermore, he could undergo generalized aches and felt a slight fever, headache, and tiredness for a day or two. Moreover, some persons sensed chills, swollen axillary lymph nodes. Hence, these sufferings denote that the body's immune system responds to the Covid shots to immunize against the coronavirus [1].

Consequently, the COVID booster shot might be from the same trademark or mixed. Moreover, the FDA, Pfizer-BioNTech, Moderna, and Janssen suggest three vaccine boosters and approved that it is unassailable to get a COVID-19 vaccine third dose even from the other trademark the initial shot. Further, the administrators ordered Sinopharm and Pfizer-BioNTech vaccines boosters safely for people aged sixteen years old [1].

It is important to note that numerous documented investigations revealed the effective broad spectrum of nanoparticles and bacteriophages as promising killer agents on viruses by inhalation. Therefore, the experimenters employed inhalational bacteriophages and nanoparticles in their functional formulations and doses to achieve an acceptable and excellent concentration on the respiratory system [4-5].

\section{Funding}

None.

\section{Conflict of Interest}

The author declared no conflict of interest for this study.

\section{References}

1. https://www.hopkinsmedicine.org/health/conditions-and-di seases/coronavirus/booster-shots-and-third-doses-for-covid19 -vaccines-what-you-need-to-know.

2. https://www.muhealth.org/our-stories/covid-19-vaccine-wh at-you-need-know-about-third-doses-and-booster-shots.

3. https://www.houstonmethodist.org/blog/articles/2021/jul/ are-covid-booster-shots-necessary/.

4. Hamad A (2021) Inhalational bacteriophage and Nanosilver Effectiveness on nCOVID 19: A Case Report. J Med Res Surg 2(3): pp. 1-5.

5. Alhiti HAR (2021) Is There Any Modern and Efficient Cure of nCOVID-19? Arch Med 13(9): pp. 1-2. 